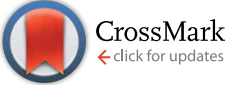

Cite this: RSC Adv., 2015, 5, 48615

Received 24th April 2015 Accepted 22nd May 2015

DOI: $10.1039 / c 5 r a 07464 a$

www.rsc.org/advances

\section{Hyaluronate-Flt1 peptide conjugate/epirubicin micelles for theranostic application to liver cancers $\uparrow$}

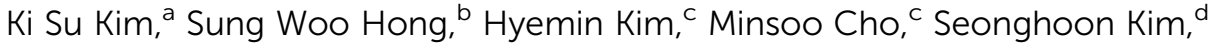

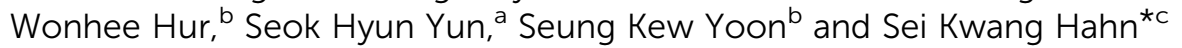

\begin{abstract}
Cancer theranostic systems using polymeric micelles are emerging for simultaneous diagnosis and therapy. In this work, we have developed hyaluronate (HA)-Flt1 peptide conjugate micelles encapsulating epirubicin for theranostic treatment of hepatocellular carcinoma. Intravital bioimaging without labelling and in vivo anti-tumor therapeutic effect clearly confirmed the feasibility of HA-Flt1 peptide conjugate/ epirubicin micelles for target-specific theranostic treatment of liver cancers.
\end{abstract}

Hepatocellular carcinoma (HCC), which is the most common type of liver cancer, ranks as the third cause of mortality worldwide. ${ }^{1}$ Liver cancer accounted for more than 800000 deaths in 2012. ${ }^{2}$ This cancer is commonly observed in patients with hepatitis $\mathrm{C}$ virus infection and liver cirrhosis. ${ }^{3}$ Liver cancer is generally treated by anti-angiogenesis and/or antiproliferation therapy via surgical resection. However, there are few clinically available drugs with a therapeutic effect on the long-term survival of patients. Recently, with the progress of nanotechnologies, a variety of nanomedicines have been developed for the treatment of liver cancer. ${ }^{4}$

Theranostic systems are currently emerging for simultaneous diagnosis and therapy. In order for these systems, micelle-like nanoparticles have been widely investigated to encapsulate a large amount of imaging agents for diagnosis and drugs for therapy within self-assembling micelles. The colloidal

${ }^{a}$ Wellman Center for Photomedicine, Massachusetts General Hospital and Harvard Medical School, 65 Landsdowne St. UP-525, Cambridge, Massachusetts 02139, USA ${ }^{b}$ The Catholic University Liver Research Center and WHO Collaborating Center of Viral Hepatitis, The Catholic University of Korea, 505 Banpo-dong, Seocho-gu, Seoul 137 701, Republic of Korea

${ }^{c}$ Department of Materials Science and Engineering, Pohang University of Science and Technology (POSTECH), 77 Cheongam-Ro, Nam-gu, Pohang, Gyeongbuk 790-784, Republic of Korea. E-mail: skhanb@postech.ac.kr; Fax: +82 54279 2399; Tel: +82 542792159

${ }^{d}$ Graduate School of Nanoscience and Technology, Korea Advanced Institute of Science and Technology, 291 Daehak-ro, Yuseong-gu, Daejeon 305-338, Republic of Korea

$\uparrow$ Electronic supplementary information (ESI) available: Experimental details and original intravital microscopic images. See DOI: 10.1039/c5ra07464a aggregation of amphiphilic molecules occurs when the concentration exceeds the critical micelle concentration (CMC). ${ }^{5}$ Micelle-like nanoparticles have been used in various drug delivery systems to increase the bioavailability of hydrophobic drugs, to reduce the cytotoxicity of drugs, to deliver small molecular drugs for a long-term, and to deliver drugs target specifically. ${ }^{6}$ Generally, hydrophobic drugs were encapsulated within amphiphilic block copolymers or conjugated to hydrophilic polymers to form a micellar structure.

In our previous work, we developed gold coated hyaluronate (HA)-doxorubicin conjugate micelles for theranostic application to cancer treatment. ${ }^{7}$ We have also developed HA-Flt1 peptide conjugate in the form of micelle-like nanoparticles. ${ }^{8} \mathrm{HA}$ is a natural polymer with the intrinsic target-specificity to the liver. ${ }^{9}$ Flt1 peptide is an anti-angiogenic hexa-peptide binding to the vascular endothelial growth factor receptor. ${ }^{10}$ HA-Flt1 peptide conjugate micelles have been used to encapsulate hydrophobic chemical drugs. ${ }^{8 c, 8 d}$

Here, we have designed HA-Flt1 peptide conjugate micelles encapsulating epirubicin (EPI) for theranostic applications to liver cancers. EPI is one of the most popular anthracycline drugs for the chemotherapy of cancers. ${ }^{\mathbf{1 1}}$ EPI causes less adverse effects, such as alopecia, dyspepsia, and myelo-suppression, than doxorubicin which has been used for cancer chemotherapy with a different chemical chirality. It has a different spatial orientation of the hydroxyl group at $4^{\prime}$ carbon of the sugar from DOX, which may account for its faster elimination and reduced toxicity. ${ }^{\mathbf{1 2}}$ Flt1 peptide and EPI in the micelle can synergistically inhibit tumor growth by the anti-angiogenesis and anti-proliferation effect.

The preparation and characterization of HA-Flt1 peptide conjugate micelles are well described in our previous reports. ${ }^{8}$ In brief, Flt1 peptide was conjugated to tetrabutyl ammonium salt of HA (HA-TBA) in dimethyl sulfoxide (DMSO). HA-Flt1 peptide conjugate was synthesized by the amide bond formation between carboxyl groups of HA and $\mathrm{N}$-terminal $\alpha$-amine groups of Flt1 peptides. Since Flt1 peptides is hydrophobic, HA-Flt1 peptide conjugate can form a micellar structure in 
aqueous solution. To encapsulate EPI in HA-Flt1 peptide conjugate micelles, we used the oil-in-water $(\mathrm{O} / \mathrm{W})$ emulsion method. The hydrophobic EPI was self-assembled into the core of micelles (Fig. 1). HA-Flt1 peptide conjugates were dissolved at a concentration of $2 \mathrm{mg} \mathrm{ml}^{-1}$ in PBS. EPI $\cdot \mathrm{HCl}$ was substituted with tetraethylamine, which was dissolved in chloroform at a concentration of $4 \mathrm{mg} \mathrm{ml}^{-1}$. The solutions of HA-Flt1 peptide conjugate and EPI were mixed at a weight ratio of $1: 2$ with a tip sonicator in an ice bath for $15 \mathrm{~min}$, and then mixed with a stirrer overnight to evaporate the chloroform completely.

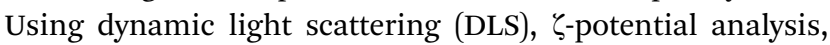
TEM and UV-vis spectroscopy, we could confirm the formation of HA-Flt1 peptide conjugate micelles encapsulating EPI (Fig. 2). HA-Flt1 peptide conjugate had absorbance at $280 \mathrm{~nm}$ due to the Flt1 peptide and EPI had absorbance at $490 \mathrm{~nm}$. HA-Flt1 peptide conjugate/EPI micelles showed absorbance at both $280 \mathrm{~nm}$ and $490 \mathrm{~nm}$. Based on the UV-vis spectra of EPI and HA-Flt1 peptide conjugate/EPI micelles, we could estimate the encapsulation efficiency to be $c a$. 35.5\%. As shown in the TEM image, HA-Flt1 peptide conjugate micelles had a spherical shape and EPI was self-assembled into HA-Flt1 peptide conjugate micelles in a core-shell structure. According to DLS, the mean particle size of micelles was estimated to be $212 \mathrm{~nm}$ with a low polydispersity index of 0.109 , which was in accordance with that in TEM image. The surface charge was $-33.9 \mathrm{mV}$ due to the negatively charged HA on the shell of the micelles.

Intravital laser scanning confocal microscopy was carried out after intravenous injection of HA-Flt1 peptide conjugate/ EPI micelles to Balb/c mice. We previously carried out the intravital confocal microscopy using HA-quantum dot conjugates with a low magnification. ${ }^{9 c}$ In this work, after cutting the abdomens of mouse, we imaged the liver using a home built microscope and a water immersion objective lens with a higher resolution $(\times 60)$. Fig. 3 shows intravital images of HA-Flt1 peptide conjugate/EPI micelles in the liver tissues. The red fluorescence of EPI in the micelles appeared mainly on the cell wall of hepatocytes within $5 \mathrm{~min}$. With increasing time, the red fluorescence was observed increasingly inside the hepatocyte, especially nucleus. The result clearly indicated that HA-Flt1

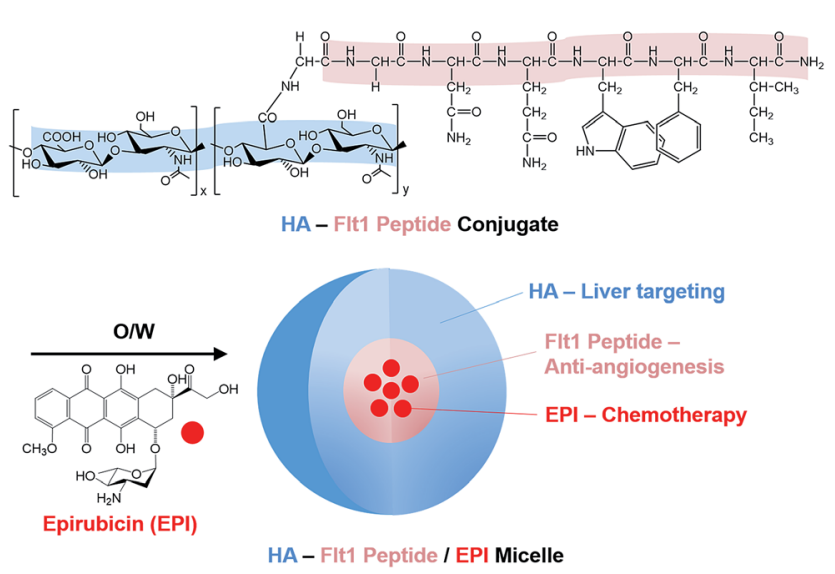

Fig. 1 A schematic illustration of the epirubicin (EPI) encapsulated hyaluronate $(\mathrm{HA})-$ Flt1 peptide conjugate micelles. (a)

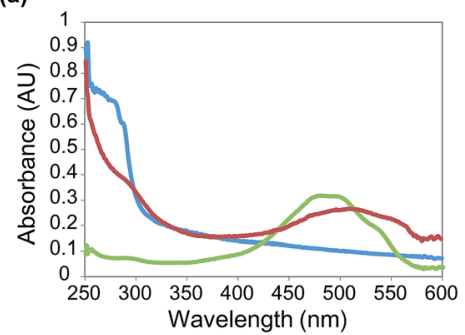

(b)

(c)
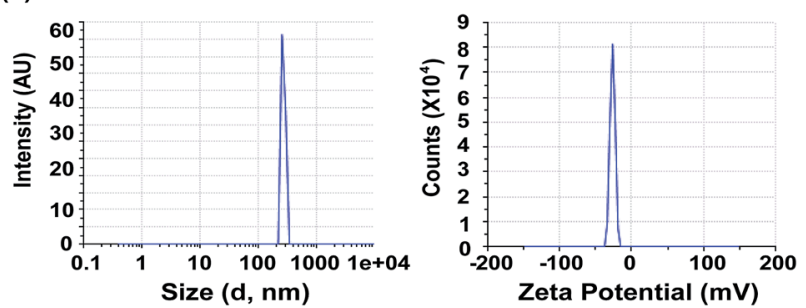

Fig. 2 (a) UV-vis spectra of HA-Flt1 peptide conjugate (blue), EPI (green), and HA-Flt1 peptide conjugate/EPI micelles (red). (b) TEM of HA-Flt1 peptide conjugate/EPI micelles (scale bars: $500 \mathrm{~nm}$ and $50 \mathrm{~nm}$ in inset). (c) DLS for size (left) and $\zeta$-potential (right) of HA-Flt1 peptide conjugate/EPI micelles.

peptide conjugate/EPI micelles were uptaken to the hepatocytes. As well known, there are abundant HA receptors (CD44 and HARE) in the liver cells. ${ }^{13}$ In addition, EPI interacts with DNA by intercalation, and inhibits DNA replication and cell division just like doxorubicin. ${ }^{\mathbf{1 4}}$ From the results, HA-Flt1 peptide conjugate/EPI micelles were thought to be accumulated in the liver cells from the blood stream via HA receptor mediated endocytosis. ${ }^{\mathbf{1 1}}$

After intravenous injection, we carried out ex vivo bioimaging to investigate the biodistribution of HA-Flt1 peptide conjugate/EPI micelles in normal and xenograft tumor mice (Fig. 4a). The tumor model mice were prepared by subcutaneous injection of B16F10 melanoma cells to the flank of C57BL/6
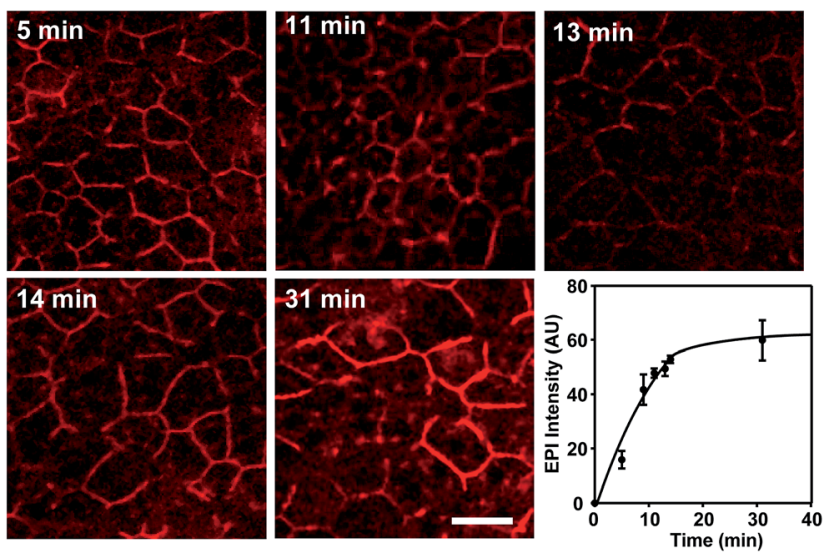

Fig. 3 Intravital microscopic images of HA-Flt1 peptide conjugate/EPI micelles in the liver tissue with increasing time post-injection. The images were normalized to have the same saturated fluorescence in blood vessels for the comparison of EPI intensity in the nucleus. 
(a)

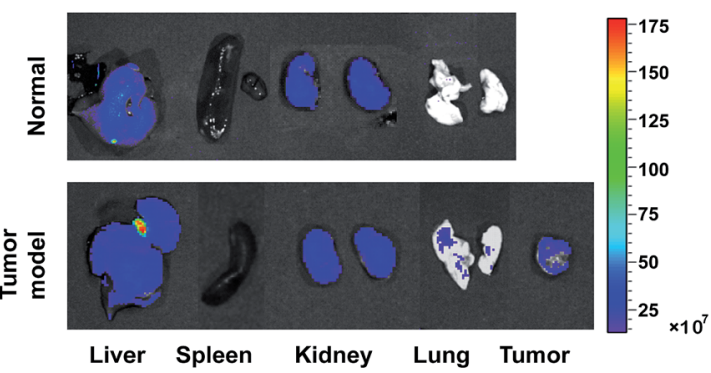

(b)

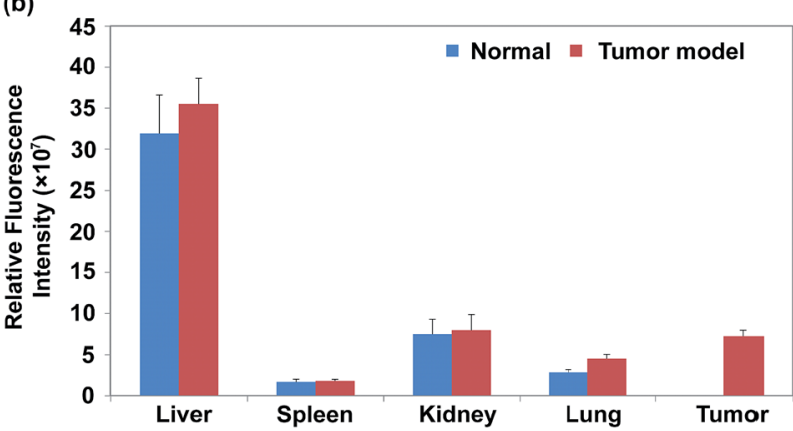

Fig. 4 (a) Ex vivo fluorescent images of HA-Flt1 peptide conjugate/EPI micelles in various dissected organs of normal and tumor model mice after 3 days post-injection. (b) Relative fluorescent intensity of HA-Flt1 peptide conjugate/EPI micelles in the dissected organs. Data are presented as means \pm SD.

mice at an age of 7 weeks. After 3 days, both mice were sacrificed for ex vivo bioimaging of their livers, spleens, kidneys, and lungs. ${ }^{15} \mathrm{~A}$ halogen lamp with a $625 \mathrm{~nm}$ filter was used for the excitation and the fluorescence images were obtained through a $790 \mathrm{~nm}$ emission filter. The fluorescence of EPI in the micelles was clearly observed in dissected liver, kidney, spleen, lung and tumor. Fig. $4 \mathrm{~b}$ shows the quantification of the biodistribution of HA-Flt1 peptide conjugate/EPI micelles in normal and tumor mice. More than half of the micelles in total organs were accumulated in the liver, and some were in the kidney in both normal and tumor model mice. Interestingly, $12.6 \%$ of the micelles in total organs still remained in tumor after 2 days post-injection. Ex vivo bioimaging clearly confirmed that HA-Flt1 peptide conjugate/EPI micelles were accumulated mainly in the liver, kidneys and tumor tissues.

On the basis of bioimaging results, we decided to assess the therapeutic effect of HA-Flt1 peptide conjugate/EPI micelles in HCC SV40T mice for theranostic applications to liver cancers. Fig. 5a shows the photo-images of the normal liver and that in HCC SV40T mice. When the tumor volume increased to 161.0 $\mathrm{mm}^{3}$ and $165.5 \mathrm{~mm}^{3}$, EPI only and HA-Flt1 peptide conjugate/EPI micelles were intravenously injected to the HCC mice at a dose of $3 \mathrm{mg} \mathrm{kg}^{-1}$ of EPI twice a week for 4 weeks. According to the magnetic resonance imaging (MRI), the tumor growth was drastically reduced by the target-specific treatment with HA-Flt1 peptide conjugate/EPI micelles without adverse effect (Fig. 5b). In contrast, after treatment with non-specific EPI, the tumor volume increased 6.1-fold $\left(984.5 \mathrm{~mm}^{3}\right)$, causing the adverse effect of alopecia and necrosis in the tail. ${ }^{8}$ (a)
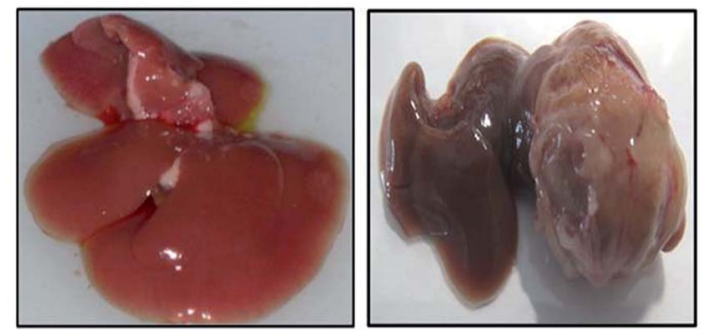

(b)

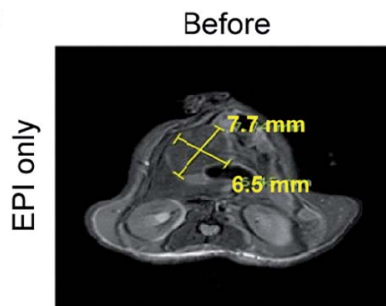

After
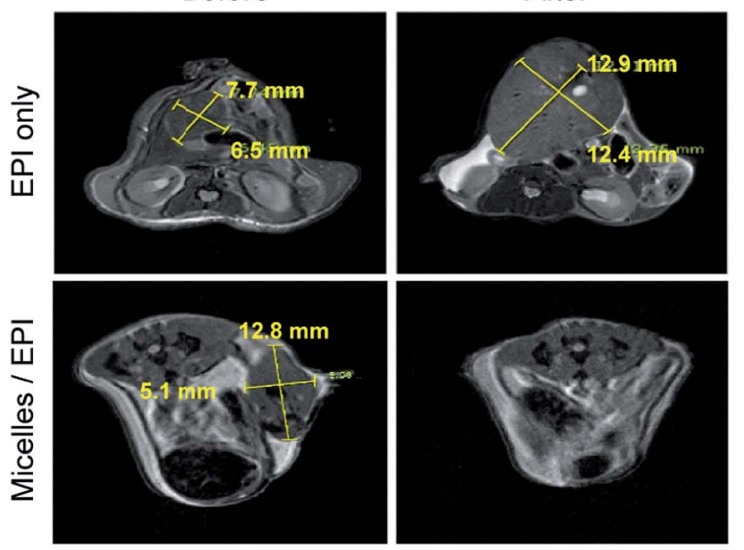

Fig. 5 (a) Photo-images of the normal liver (left) and that in hepatocellular carcinoma (HCC) SV40T mice (right). (b) Magnetic resonance imaging of $\mathrm{HCC}$ tumor tissues before and after treatment with EPI only or HA-Flt1 peptide conjugate/EPI micelles for 4 weeks.

On the basis of this preliminary study, we will carry out the preclinical study on the therapeutic effect of the micelles using a large number of animals. All these results confirmed the feasibility of HA-Flt1 peptide conjugate/EPI micelles for theranostic applications to liver cancers.

\section{Conclusions}

We successfully carried out the theranostic application of HA-Flt1 peptide conjugate/EPI micelles to the treatment of liver cancer. The formation of micelles and the encapsulation of EPI were confirmed by UV-vis spectroscopy, TEM, and DLS analysis. In vivo and ex vivo bioimaging clearly visualized the targetspecific delivery of the micelles to the liver. Finally, we could confirm the therapeutic effect of the micelles from the significantly reduced tumor growth. Taken together, HA-Flt1 peptide conjugate/EPI micelles can be effectively exploited for theranostic applications to liver cancers.

\section{Acknowledgements}

This research was supported by a grant of the Korean Health Technology R\&D Project, Ministry of Health and Welfare, Korea (HI14C1658) and the Bio \& Medical Technology Development Program (no. 2012M3A9C6049791) of the National Research Foundation (NRF) funded by the Korean government (MEST). 


\section{Notes and references}

1 T. W. Kensler, G.-S. Qian, J.-G. Chen and J. D. Groopman, Nat. Rev. Cancer, 2003, 3, 321.

2 World Health Organization, in World Cancer Report 2014, ed. B. W. Stewart and C. R. Wild, IARC Publication, Lyon, 2014, ch. 1.1, p. 16.

3 P. A. Farazi and R. A. DePhino, Nat. Rev. Cancer, 2006, 6, 674. 4 M. Ferrari, Nat. Rev. Cancer, 2005, 5, 161.

5 (a) R. Duncan, Nat. Rev. Drug Discovery., 2003, 2, 347; (b) C. Burda, X. Chen, R. Narayanan and M. A. El-Sayed, Chem. Rev., 2005, 105, 1025.

6 (a) B.-S. Kim, S. W. Park and P. T. Hammond, ACS Nano, 2008, 2, 386; (b) N. Nasongkla, E. Bey, J. Ren, H. Ai, C. Khemtong, J. S. Guthi, S.-F. Chin, A. D. Sherry, D. A. Boothman and J. Gao, Nano Lett., 2006, 6, 2427.

7 K. S. Kim, S.-J. Park, M.-Y. Lee, K.-G. Lim and S. K. Hahn, Macromol. Res., 2012, 20, 277.

8 (a) E. J. Oh, K. Park, J.-S. Choi, C.-K. Joo and S. K. Hahn, Biomaterials, 2009, 30, 6026; (b) E. J. Oh, J.-S. Choi, H. Kim, C.-K. Joo and S. K. Hahn, Biomaterials, 2011, 32, 3115; (c) H. Kim, H. T. Park, Y. M. Tae, W. H. Kong, D. K. Sung, B. W. Hwang, K. S. Kim, Y. K. Kim and S. K. Hahn, Biomaterials, 2013, 34, 8478; (d) H. Kim, J. S. Choi,
K. S. Kim, J. A. Yang, C. K. Joo and S. K. Hahn, Acta Biomaterialia, 2012, 8, 3932.

9 (a) K. S. Kim, W. Hur, S.-J. Park, S. W. Hong, J. E. Choi, E. J. Goh, S. K. Yoon and S. K. Hahn, ACS Nano, 2010, 4, 3005; (b) E. J. Oh, K. Park, K. S. Kim, J. Kim, J.-A. Yang, J.-H. Kong, M. Y. Lee, A. S. Hoffman and S. K. Hahn, J. Controlled Release, 2010, 141, 2; (c) K. S. Kim, S. Kim, S. Beack, J.-A. Yang, S. H. Yun and S. K. Hahn, J. Nanomed. Nanotechnol., 2012, 8, 1070.

10 D. G. Bae, T. D. Kim, G. Li, W. H. Yoon and C. B. Chae, Clin. Cancer Res., 2005, 11, 2651.

11 R. J. Cersosimo and W. K. Hong, J. Clin. Oncol., 1986, 4, 425. 12 M. Bontenbal, M. Andersson, J. Wildiers, G. Cocconi, J. Jassem, R. Paridaens, N. Rotmensz, R. Sylvester, H. T. Mouridsen, J. G. M. Klijn and A. T. van Oosterom, $B r$. J. Cancer, 1998, 77, 2257.

13 (a) S. Banerji, A. J. Wright, M. Nobel, D. J. Mahoney, I. D. Cambell, A. D. Day and D. G. Jackson, Nat. Struct. Mol. Biol., 2007, 14, 234; (b) J. A. Weigel and P. H. Weigel, J. Biol. Chem., 2003, 278, 42802.

14 O. Tacar, P. Sriamornsak and C. R. Dass, J. Pharm. Pharmacol., 2013, 65, 157.

15 E. J. Goh, K. S. Kim, Y. R. Kim, H. S. Jung, S. Beack, W. H. Kong, G. Scarcelli, S. H. Yun and S. K. Hahn, Biomacromolecules, 2012, 13, 2554. 